\title{
A TAXONOMY OF WEB AND WINDING DEFECTS
}

By

\author{
David R. Roisum, Ph.D. \\ Finishing Technologies, Inc. \\ USA
}

\begin{abstract}
Constructing taxonomies can be useful for the researcher and troubleshooter alike. First, it gives us a precise language for conversation within our own plant or with our customers. This language helps us avoid using the same word for two different things or using two different words for the same thing. Examples of word confusion are corrugations versus tin-canning as well as telescoping and coning. The former pair are two distinctly different defects, while the latter pair are aliases for the same defect class. Second, taxonomies allow us to make crucial distinctions between similarly appearing defects, but whose mechanics and thus remedies may be quite different. Examples include a wrinkle oriented at an angle means something (roller, web etc) is 'crooked,' while the root cause mechanics for a wrinkle oriented in the MD may have width related mechanics. The former might indicate the need for better roller maintenance while the latter might indicate the need for a spreader.

As useful as good taxonomies might be, they can be exceedingly difficult to do well. Very often they are a work in progress. They are always part craft and part science. On the craft end, we might offer as an example the troubleshooting trees used by automobile mechanics to identify which part needs to be adjusted or replaced to get an engine running. On the science end we might offer the classification of life forms which used to be based entirely on form, but is now occasionally assisted by DNA comparisons, when it is available.

In this paper we will show how to construct taxonomies for some common defects. While good taxonomies are mechanics based, these mechanics and the attendant complexities should be hidden from the user to the extent possible. The goal is to be able to do most everything observationally rather than requiring equations or instruments because it greatly increases the utility of the system. However, the researcher can also use a good taxonomy if it is mechanics based. In any case, everyone can benefit from a precision of language. The defects we will include here are most forms of web flatness/wrinkling as well as some winding defects such as core crush, starring and telescoping.
\end{abstract}


KEYWORDS Taxonomy; classification; troubleshooting_tree; wrinkle; wind; defect

\section{MOTIVATION}

Constructing taxonomies can be useful for the researcher and troubleshooter alike. It gives us a precise language for conversation within our own plant or with our customers. This language helps us avoid using the same word for two different things. An example is 'corrugation,' that can be used for both a true corrugation as well as an alias for a 'tin can' as some film people are wont to. Yes, both are 'tight' winding defects, but both are distinctly different in mechanism and have different remedial options. A good taxonomy also helps avoid using different words for the same thing. Thus, when someone complains of 'coning' or 'shooting' or 'scoping', you know they are probably referring to one of the half-dozen telescope-like defects. Unfortunately, there are many distinctly different telescope defects that look roughly similar. A good taxonomy can make the distinctions between similarly appearing defects, but whose remedies may be quite different

As useful as good taxonomies might be, they can be exceedingly difficult to do well. Very often they are a work in progress. Nearly always they are both a craft and a science. On the craft end we might offer the troubleshooting trees used by automobile mechanics to identify which part needs to be adjusted or replaced to get an engine running. On the science end we are familiar with the classification of life forms. Perhaps a thousand years ago it might have made sense to group whales and sharks together as ocean fish, or bats and birds together as flying animals. When we were in school, there were five life kingdoms: animals, plants, fungi, algae and prokaryotes. Now there are three kingdoms. DNA and evolutionary biology have rearranged the details of the branches many times and there is still much dispute and reclassification occurring even today. The matter is still not settled and will never be settled even using DNA because people may prefer 'lumping' to 'splitting' or vice versa. Still, no one can argue how essential the taxonomic tree has been to the life sciences.

In this paper we will show how to construct taxonomies for some common defects. While good taxonomies are mechanics based, these mechanics and the attendant complexities should be mostly hidden from the user. The goal is to be able to do most everything observationally rather than requiring equations or instruments because it greatly increases the utility of the system. However, the researcher can also use a good taxonomy because it is mechanics based. No matter what role we work in, everyone can benefit from a precision of language. The defects we will include here are most forms of web flatness/wrinkling as well as some winding defects such as core crush, starring and telescoping.

\section{A SHORT HISTORY OF WEB AND ROLL DEFECT CLASSIFICATION}

While not called taxonomies, many early papers and pamphlets and books on defects functioned as such. The earliest probably came from newsprint printing. This should not be surprising because paper is the oldest of the (non-textile) webs and newsprint was at the time the biggest of the paper grades. A very good example is an obscure but welldone pamphlet on newsprint defects written by a large paper company [1]. Ken Frye, of the former Beloit Corporation winder division, went to newsprint printing houses in a rare early outreach from (paper mill) winders to try to understand the needs of their 
customer. This resulted in a series of articles in 1986 such as [2]. Coating is the second largest converting segment (after printing) and thus, not surprisingly, also a subject for early defect classification systems as both a book [3] and later a website [4]. By far, the most ambitious defect classification system was first published by TAPPI in 1973 as a CA (committee action) as a pamphlet [5] and then 4 generations later in 2014 as a 500 page encyclopedic treatment [6]. Of course, there are many, many articles and conference papers with a much narrower scope of coverage such as telescoping [7] and wrinkling [8].

\section{THE STRUCTURE}

The structure of a taxonomy or tree is, well, a tree. Here we will use a word outline instead of the more graphic tree simply for compactness. Specifically, the structure will look like this:

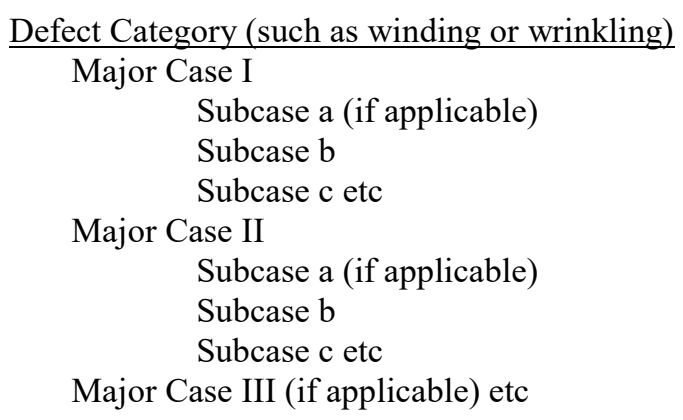

So in the Defect Category of wrinkling, for example, all flatness and wrinkles of any cause or flavor share the same outcome. The web is not perfectly flat, due to in-plane compressive stresses in most cases. We can break this wrinkling defect category into about a half dozen different Major Cases. A Major Case shares the same mechanics. An example is the major case of MD wrinkles where the wrinkle (trough, bump on roller, foldover, crease etc) orientation is very close, perhaps within 2 degrees, of the Machine Direction. There are nearly a dozen subcases of MD wrinkles at this time and each has distinct root cause mechanics, most related to excess width. On the other hand, the Defect Category of core crush has three major cases at this time, but there are no subcases. The root cause mechanics are the three major cases.

A few notes about the tree structure before we begin. Each case or subcase should be mutually exclusive and the cases should cover most or all cases. To be mutually exclusive means that there needs to be an unambiguous test to determine which branch of the tree you are on. To be very useful, this test should be simple and observational rather than requiring special measurement tools, equations or numbers. There are, of course, several limitations to this approach. First is that most defects simply do not lend themselves to this approach at this time because we either don't know the subject well enough, aren't creative enough to see the tree, or that a useful test for which branch we are on has not been found yet. Still, many problems lend themselves quite well to a welldesigned taxonomy. Second, often more than one root cause mechanics is involved at a specific location on a single web. For example, you could have both a diagonal wrinkle on the side of a baggy lane as well as a diagonal wrinkle due to roller misalignment. They both could be present in the same (MD location in the machine or cut specimen) place and at the same time. Still, a good tree will be able to separate the distinct causes and 
guide the troubleshooter to the appropriate remedy options for both. It is sometimes possible to have the same remedy option to be effective for two distinct root cause mechanics. Spreading is one example, though it is more effective with some types of wrinkles, such as MD wrinkles, than others, such as diagonal wrinkles. Web tension and winding tightness are other examples. You could have two distinctly different 'tight' defects, such as bagginess and corrugations, in the same wound roll. If so, some relief for both might be achieved by reducing the nip for example. Still, these complications and other can easily be accommodated by a taxonomic procedure.

\section{CORE CRUSH}

Let us begin with a simpler defect because it has fewer branches and because determining which branch you are on is easy. Here we will focus on core crush knowing quite well there are many other important core failure modes such as axial failure, loose cores (due to drying out of fiber cores after winding), loose fits of cores on chucks/shafts and many more. Core crush, the defect category, can be defined quite simply as a large compressive deformation of the core during or after winding. There are three major cases corresponding to three quite distinct load cases as summarized in Figure 1. Case I is most common when winding low modulus materials such as rubber, nylon, PE and PP. Here internal wound-in-tension readily translates to radial compression that can exceed the strength or buckling strength of the core. This case is, obviously, a tight defect. A further observation that can determine the major case is that the crush occurs during or shortly after winding. Case III is most common with the winding of high modulus materials such as paper, paperboard and metal. Here it is external loads such as coming from roll handling, such as clamp trucks, that pass through all the layers to the core and subsequent failure. This defect is totally different because loads that crush the core come from outside the roll, not inside. This defect is totally different because the core crushes not during winding, as in Case I, but rather during handling. Finally, this defect is totally different because it is, perhaps surprisingly, a loose defect. Tight winding helps make the layers of the roll act like arches to protect the core from outside forces. Case II is far less common, but the load case is chemical and/or thermal shrinkage. BOPP (Biaxially Oriented PolyPropylene) is the exemplar for this type of misbehavior. This is a different defect because the loads that knocked out the core do not come from winding or the outside world, but rather from chemistry. While this is strictly speaking a tight defect, in some web chemistries or conditions the stresses coming from the material are larger or much larger than those coming from normal winding. When chemical/thermal contributions are significantly larger, winding looser, even as loose as practicable, may not have a significant benefit.

The core crush defect nicely illustrates the option list. First, we would start with the winder by winding either tighter or looser depending on the major case that is specific to our problem and by using any and all of the TNT (Tension, Nip, Torque and other) controls to the extent possible. Further gains would require redesigning the product, namely the core. Figure 1 illustrates these different major cases of core crush and the product redesign options that are common to most or all of the major cases. Still, this troubleshooting tree does not include a few of the rarer core crushes. One example is the 'sag collapse' of large coils of metals as shown in Figure 2. While most closely related to Case III above, the loads for gravity are different enough from clamp truck squeeze that it might be given a separate case. 


\section{STARRING}

In some ways starring is similar to core crush. In fact, when the core crushes a star will also result so that is one of the major cases. Starring is also similar because we have a compressive buckling failure, but this time it is the layers of the roll instead of the core. So, as we should come to expect, starring is not a single defect but a collection of distinctly different defects that have the same outcome; buckling of inner layers in a wound roll. Unlike core crush, however, we have more major cases and now for the first time in this discussion, subcases. The troubleshooting tree below organizes and distinguishes the cases.

$\underline{\text { Starring Troubleshooting Tree }}$

I Poor Roll Structure

A Insufficient 'Taper'

B Winder Stop

C Upset in TNT controls

II Unsupported Layers

A Roll offset/dish/telescope

B Core narrow or inset

C Core collapse

D Air Buckle (escape of air out edges)

III Thickness Profile Variation

A Intentional (e.g., not coating/printing to edges)

B Unintentional (poor thickness profile control)

IV Rough Handling
A Blow
B Squeeze
C Etc

Looking briefly at some of the cases we see, for example, that for Case 1 Poor Roll Structure the subcases have to do with winding TNT controls as the dominant factors. Note also that we could have included thickness profile under unsupported layers, but chose not too because the dominant remedy is winding controls in one major case and manufacturing/converting in another. Similarly, we could have including rough roll handling under Poor Roll Structure and relabeled that major case as Insufficient Roll Structure. Discussions such as nicely illustrate the art/craft/judgment in how to slice and dice categories. While the purest might balk as such discretion, science rarely tells us how to do taxonomies. The rare exception might be constructing the tree of life strictly as a function of DNA progression through time, but that is well beyond the state of biology even at this time.

\section{TELESCOPING}

The defect category telescoping is when roll edges smoothly change from mostly flat/straight to some other shape usually during winding or unwinding, and in one case during storage [7]. The telescoping defect is different than rough roll edges, which we will do next because telescoping is a disturbance on a large scale and rough roll edges on a much finer scale. 
Again, as we should come to expect, telescoping is not a single defect but rather a collection of mostly unrelated defects that have the same outcome; smoothly varying roll edge shape.

\section{$\underline{\text { Telescoping Troubleshooting Tree }}$}

I MD Interlayer Slippage (that precedes CD slippage)

A During Winding

B During Unwinding

II CD Slippage during storage

A Adhesives that flow (such as PSA's)

B Foam/Nonwoven/Textile creep of narrow aspect ratio wound rolls III CD Slippage during handling

A Axial lift

IV Progressive Outward Dishing (two drum winder roll width growth) $\mathrm{V}$ Other

A Clockspringing - unraveling of slippery rolls, such as $35 \mathrm{~mm}$ film)

B Inertial Clockspringing - slowing down of a finished roll by core torque

C Axial movement of the core chucks or core shaft during winding

Here we will focus on the most common major case, that is MD interlayer slippage during the latter stages of core supported winding, subcase A, and slippage during the initial stages of unwinding, subcase B. What is common is that torque applied to the core causes layers to slip first in the MD that then allows slippage in the CD. Figure 3 illustrates the principals and test for this most common case. The mechanism and litmus test for this is to stop the machine midway during the wind, strike a radial line and resume. This is only done under when strict LOTO (Lock-Out-Tag-Out) and other safety protocols can be followed. The wind is then resumed. When the roll is finished winding you will see the layers near the core have indeed slid. A supporting observation is simpler to do, but is not always unambiguous. That is the classic roll edge shape may show a curve-straight-curve pattern. The curve above the core is the layers that slid. The straight intermediate section is layers moving outward as a solid body as they ride out on the sliding layers beneath. The top section is also a curve, the bottom of which shows you when the roll started to slide at the bottom. A final supporting observation is that the outside of the roll edges line up with the core because that is where those layers were applied and those layers did not slide. Once the diagnosis is thoroughly confirmed with the litmus test and supporting observations, you can be confident in listing remedy options as below in Table 1 .

Maximum Tension Taper, Maximum Nip and Torque at bottom of roll.

Increase Core Diameter

Decrease Roll Diameter

Side Guards

Reduce storage time and/or unwinding torque for case IB

Table 1 - Telescoping IA Remedy List

Note that the unwinding case is almost identical in all relevant aspects including the remedial list. A picture of Telescoping 1B is shown in Figure 4. One should be wary that 
just because you can wind a roll does not mean you can unwind that same roll. The torques applied may be larger, the fiber core might have shrunk or the material might have gotten slipperier during storage as we know many papers, films, inks, and coatings are wont to do. Since the world is complicated, I will just note that nonwovens and other bulky materials can behave almost precisely the same, and the mechanism is still MD interlayer slippage that allows CD slippage, but the MD slippage is due to the weight of the 'nip' of the roll above the core rather than slipperiness.

Telescoping Case II is nearly pure CD slippage that is enabled by lubrication of layers. PSA's (Pressure Sensitive Adhesives) are the poster child of this problem. Note however that other materials can creep and thus give nearly identical behavior. It is interesting to note the differences between Type I and Type II telescoping as given in Table 2 . This clearly illustrates why diagnostic trees and tests and precise language is needed. Probably the most salient is that a Type I telescope is a tension taper defect while Type I is a tight defect. After the winder TNT's are moved in the correct direction to the extent possible, one could consider product redesign. In Type I it would be roll/core sizes and/or web-web COF. In Type II it would be roll aspect ratios (tall is more risky) and the shear viscosity of the adhesive if that were the 'lubricant' which also implies keeping rolls out of high temperature storage for long periods. Not that in some extreme situations, it is possible to have a Type I and Type II telescope at the same time. Here, it is clear that the ending tightness should be minimum. However, the starting tightness is some compromise between the two telescopes. Usually, however, the product must be redesigned as this situation may be well beyond winding remedy.

\begin{tabular}{|c|c|c|}
\hline Observation & Type I & Type II \\
\hline Slippage occurs & $\begin{array}{c}\text { During } \\
\text { winding/unwinding }\end{array}$ & During (hot) storage \\
\hline J-Line & Yes & Little or none \\
\hline $\begin{array}{c}\text { Classic Roll Edge } \\
\text { Shape }\end{array}$ & $\begin{array}{c}\text { Curve-straight-curve } \\
\text { (crater with central cone) }\end{array}$ & Bowl or bullet \\
\hline $\begin{array}{c}\text { Best TNT Program at } \\
\text { bottom, middle and top of } \\
\text { roll }\end{array}$ & $\begin{array}{c}\text { Tension = max, max, } \\
\text { min Nip = max, -, - }\end{array}$ & $\begin{array}{c}\text { Tension = min, min, } \\
\text { Nip = min, min, min }\end{array}$ \\
\hline
\end{tabular}

Table 2 - Comparison of Type I and Type II Telescopes

We will not discuss most other telescope types, of which there are several. They are far less common and quite a bit more involved to describe via words, pictures or mechanism. An example of a rarer type is the progressive outward dishing when many rolls run side by side on a wide two drum winder. Another is the uncoiling of slippery materials such as $35 \mathrm{~mm}$ photographic film, rolls of postage stamps and coils of sheet metal when the tail is untacked from the roll.

\section{ROUGH ROLL EDGES}

The edges of a roll on a fine scale might be thought of like (deciduous) tree rings. A narrow ring could indicate a season that is too hot, too cold, too short, too wet, too dry or that the tree was plagued with fungi or insects. Thus, one would know that that season was different, but that not necessarily the mechanism by observation of the ring narrowness alone. The edge of the wound roll is in many ways analogous. It can show many things that you did wrong either on the winder or upstream in manufacturing. Since 
there are so many possibilities of material, mechanism, winder and winder settings, we will just get started with the tree structure and let the practitioner fill out the rest.

Cleverness coupled with specially created tests would tell you on which branch of the tree you were on. For example, peeling down to the single layer being studied you could easily tell whether the web width changed or (web or roll) position changed merely by careful ruler (or optical comparator) measurements.

$\underline{\text { Rough Roll Edges on a Fine Scale }}$

I Web Moved

A Upstream of windup

B Interlayer slippage at very top of winding roll

II Roll Moved

A Axaily loose core fit

B Frame moved

III Web Changed Width

A Raw material width changed

B Tension changed (at slitters or winder)

C Poisson ratio changed

D Slitter moved

One notes that for this defect, a tree structure would be very difficult to create in any kind of completeness. Organizing a tree to include other sources of roll edge disturbance such as air entrainment, vibration or oscillation rings (shown by progressively decreasing ring spacing) would be something perhaps the next defect author might like to try.

Still, the concept of a binomial search strategy as we just used for rough roll edges may also be adapted to 'loss of trim,' because many of the elements are the same or similar.

\section{$\underline{\text { Loss of Trim }}$}

I Web Moved Upstream of Slitter

II Trim Chute Moved

III Web Changed Width

A Raw material width changed

B Tension changed (at slitters or winder)

C Poisson ratio changed

D Slitter moved

\section{WRINKLING AND FLATNESS}

Wrinkling and flatness may be the number one source of waste, delay and customer complaints in the web industry overall. Thin paper grades are plagued by chronic flatness problems when running through machinery, on winding rolls and upon unwinding. The resulting paper flatness problems range from discussion to complaint to web breaks. Wrinkling is also in the top three troubles for most film plants. Sometimes flatness issues get a different label and thus disguises mechanism. For example, color-to-color registration in printing is one of their top concerns and registration is degraded when running baggy webs and baggy webs are, well, a flatness problem. Crooked printing 
equipment can announce itself as a lack of perfect web flatness, but more importantly can also contribute to poor color-to-color registration. No matter what rank wrinkling and flatness has in your plant or your customer's plant, it probably causes enough trouble to deserve taxonomic and diagnostic effort.

The primary problem in getting started in wrinkling is probably conceptual. People get in debates as to whether a wrinkle is 'bad enough' to reject or to be called a wrinkle. Some people's 'pucker' is tolerable until it folds over while others will reject far earlier. People get in debates as to whether bagginess is a wrinkle or something else. People could debate why curl, for example, should be lumped together with more classic wrinkles such as the diagonal shear wrinkle caused by excessive in-plane roller misalignment. People would observe that both bagginess and misalignment cause diagonal features, call them wrinkles for now, and wonder what is in common. The salient question arises, "how do you bring all of these and many other issues together in a world of such diverse material chemistries and such diverse machinery?"

The answer is, by treating all forms of less than perfect flatness as a 'wrinkle.' We will let the customer or QA determine the threshold of pain appropriate for them. We as troubleshooters want to know why the web is not dead flat (or flat enough). The simple answer is that all forms of flatness issues, call them wrinkles just for compactness, have one other thing in common: in-plane compressive stresses. Our job is to find out what made those compressive stresses, the 'root cause mechanism' in common parlance, which then leads us directly to a remedy list to consider. In short, a wrinkle is not a wrinkle is not a wrinkle. Each type, or subtype in this case, has a specific set of factors that are relevant. What is relevant are certain web physical properties such as width, thickness and modulus. What is almost irrelevant is web chemistry except as how it affects modulus and other properties. Same with machine factors. Precisions matter while nameplate, vintage and type not so much.

We will refer to Table 3 for the following discussion. The first breakdown of the wrinkling defect category (where all subtypes are lack of flatness) are the major classes including orientation of the wrinkle feature (MD, Diagonal and CD). The first breakdown also includes other types not so conveniently described by angle such as baggy web, corrugations and curl. Note that while bagginess and corrugations will have features at an angle, they are distinctive enough in mechanism to not include them in the diagonal major class. This is the art of taxonomy to know when diagnostics may be hindered by hewing to a too strict discipline of mechanics, in this case shear stresses that are common to all. Regardless, the major cases in the tree shown here meets one of our primary requisites, simplicity. Anyone can at a glance determine which major case they have. No special tools or training are required.

\section{MD Wrinkle}

A Tension too high (stretchy materials)

B Tension drop in span (stretchy materials)

C Temperature increase (film, foil)

D Moisture increase (nylon, paper)

E Slender roller deflection

$\mathrm{F}$ Excessive roller grooving width

G Roller groove or bump

$\mathrm{H}$ Lane in raw material

I Improper spreading (or a slight outward fan) 
II Diagonal Wrinkle

A. Crooked Machine

\author{
a. Roller misalignment \\ b. Roller cylindricity profile \\ c. Nip uneven, etc, etc, etc \\ Crooked Web \\ d. Bagginess (separate major category) \\ III. CD Wrinkle \\ e. Skew of principle axis w.r.t MD, etc, etc \\ A. Air and other Buckles at bottom of roll \\ B. Delamination of loosely bonded plies of a laminate \\ IV. Baggy Web \\ A. Formed baggy \\ B. Stretched over gage bands on winder \\ V. Curl \\ A Manufacturing Curl (one sided formation) \\ B Converting Curl (such as coating on one side) \\ C Laminator Curl (mismatch of strains at bond point) \\ D 'Roll' or Core Set Curl (due to creep in a roll)
}

Table 3 - Wrinkle / Flatness Troubleshooting Tree

Considering the MD wrinkle major case, we note that all subcases, root causes if you will, have a common mechanism related to web width as defined by the distance between its two edges. As you can see, the MD wrinkle has a lot more subcases than the winding defects that we discussed earlier. Still, each shares the MD orientation and most relate in some way to width. Also, most would be amenable to spreading, though some much more than others. Moving on to the Diagonal wrinkle we note that all subcases result in a wrinkle and an angle and have to do with shear stresses in mechanics language. However, in layman's language, something, machine or material, is crooked. We can distinguish which, machine or material, owns the crookedness via any number of cleverly constructed tests. Probably the easiest (in most cases) and most decisive is to flip the unwinding roll end for end (top for bottom) in the middle of a run as seen in Figure 5. There are other ways both decisive or merely indicative to determine whether the issue is largely machine or material [7]. If the defect moves with the material, it is the material and vice versa. Unfortunately, there are so many ways to make things, machine or material, crooked that listing them here would not be practical. Describing them would be out of the question. Still, this taxonomy provides a template for a structured language and view so that new forms of crookedness can be easily added as they are found and precisely described. $\mathrm{CD}$ wrinkles have, at least on this writing, only two subcases: certain buckles on the bottom of the wound roll, such as most typified by the air buckle, and delamination sometimes called 'tunnel' wrinkles. We will shorten this discussion to end with curl. As should be anticipated, all are connected by having MD tensile stresses that are different on the top compared with the bottom. As should be anticipated, all have a similar appearance or outcome. As should be anticipated, each of the subcases has a different root cause mechanics and remedial option set, though some remedies may overlap. One sided forming, lamination and roll-set curl are entirely different in mechanism and have largely different sets of factors that can be manipulated to reduce the problem. Note that we can counter one type of curl, say laminating curl, against another, say roll set curl, by choosing which side to wind in. While this may not be 
allowed by the customer is a different discussion, making lists based on the specific mechanics of the problem and choosing are never to be mixed. This option of countering is also not perfect as rollset curl varies with distance above the core while laminating curl does not.

\section{SUMMARY AND FUTURE WORK}

As we saw, taxonomy is essential for precision of language. Though troubleshooting trees are extremely difficult to construct, if done well they would help everyone from the operator to the $\mathrm{PhD}$. Most practically, they tell us what options are helpful for specific defects or groups of defects. Still, taxonomies are not carved in stone. They are a work in progress. Future work may rearrange branches and hopefully add many more. As an example, we adapted the rough roll edge taxonomy to work on a totally different type of problem: 'loss of trim,' because many of the elements were the same or similar.

The taxonomy approach does have its limitations. As we saw, listing the number of ways that a machine or web could be 'crooked,' all producing a wrinkle at an angle, would be daunting and probably quite unnecessary. The practitioner can take those last steps in the field. If they had a product with an intentional patterned edge cutout and a diagonal wrinkle appeared nearby, they would with a bit of liberal thinking recognize the aspects of crookedness belonged to product design. Another very well studied defect, brittle web breaks, would probably not be helped with a troubleshooting tree [9]. At best we would end up with a list of risk factors to check for, a process that is already in common use.

Still, no tool can be expected to be useful in all cases [10]. That is why experts and practitioners alike develop and collect as many tools as practicable. It increases the chance that one tool will fit better or fit well or that more than one tool can be used on the same problem thus increasing confidence in the solution.

\section{REFERENCES}

1. Percival, A. A., About Newsprint, Smurfit Newsprint Corporation, 1994.

2. Frye, K. G., "Problem Solving in the Finishing Room," TAPPI Journal, Vol. 69, No. 3, March 1986, pp. 108-112.

3. Gutoff, E. B., Coating and Drying Defect Problems - Troubleshooting Operating Problems, John Wiley \& Sons, Inc., 1995.

4. Cohen, E. D., "Coating Defects Dictionary," AIMCAL Fall Tech. Conf., Rock Hill, SC, October 26-29, 2003.

5. Gilmore, W. G., "Report on Roll Defect Terminology," TAPPI CA 1228, TAPPI Finishing and Converting Conf. Proceedings, Atlanta, GA, TAPPI PRESS, 1973.

6. Smith, D. R., Ultimate Roll and Web Troubleshooting Guide. TAPPI PRESS, 2013.

7. Roisum, D. R., "Telescoping of Adhesive Coated Wound Rolls," TAPPI PLACE Conf. Proc., Boston, September 9-12, 2002.

8. Roisum, D. R., “The Mechanics of Wrinkling," TAPPI Journal, Vol. 79, No. 10, October 1996, pp. 217-226.

9. Roisum, D. R., "Runnability of Paper," TAPPI Journal, 1990.

10. Roisum, D. R., Critical Thinking in Converting, Atlanta, GA: TAPPI PRESS, 2002. 


\section{Core Crush}

- Adjust Wound-in-Tension First

I. Looser if crush during winding (e.g. film)

II. Looser if crush due to material shrink (rare)

III. Tighter if crush during handling (e.g. paper)

- Redesign Cores Next

- Wall thickness increase (quite powerful)

- Core diameter decrease (if customer allows)

- Core plugs (narrow rolls Cases II \& III)

- Stronger core (using better grade of fibers)

Figure 1 - Three Core Crush Cases and Options

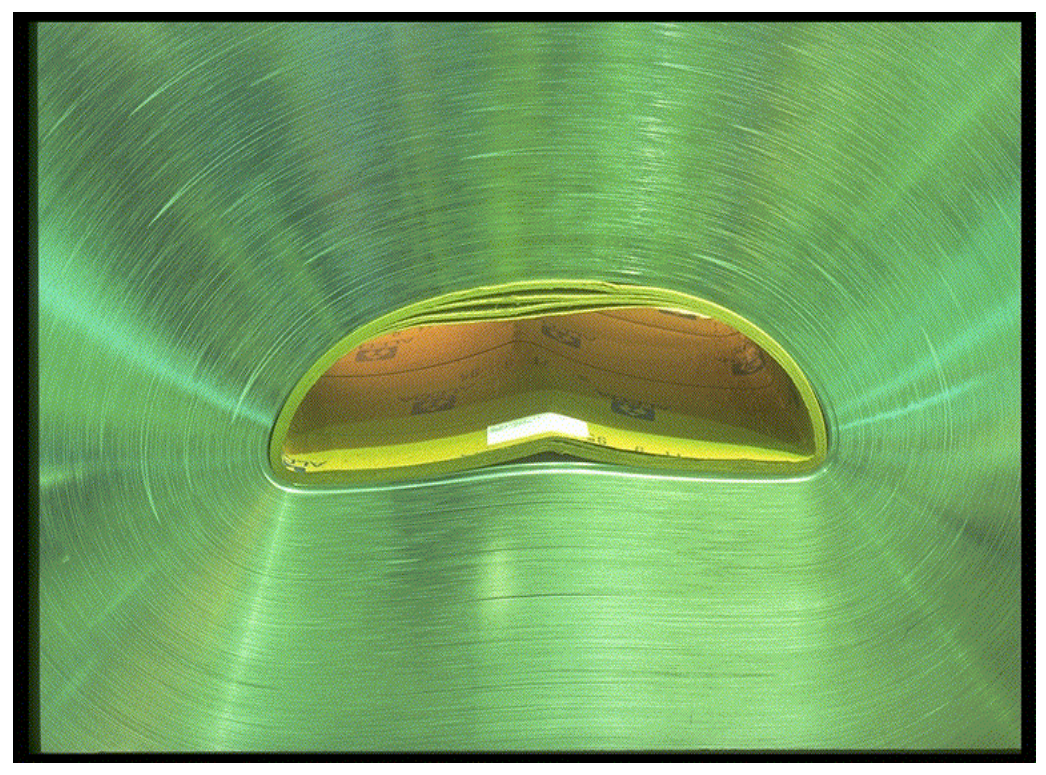

Figure 2 - 'Sag Collapse' of a Large Coil of Metal 


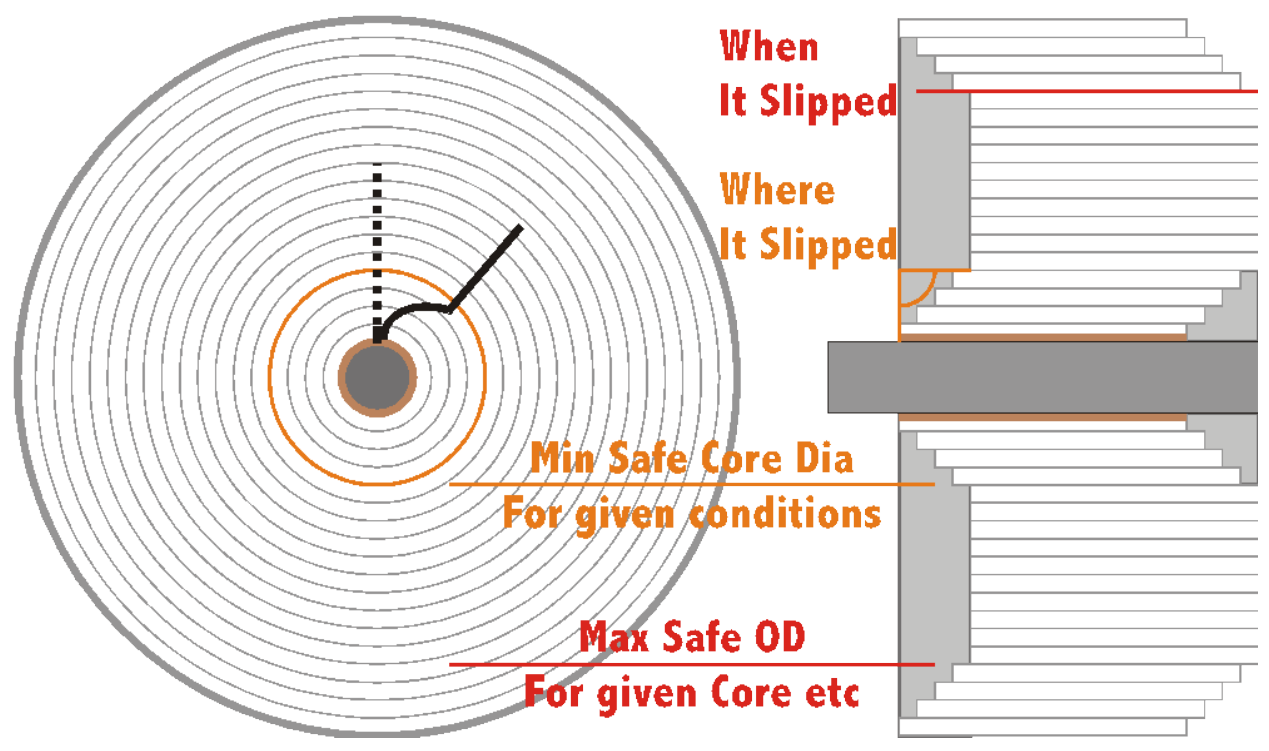

Figure 3 - Diagnosing Type 1A (Winding) Telescope

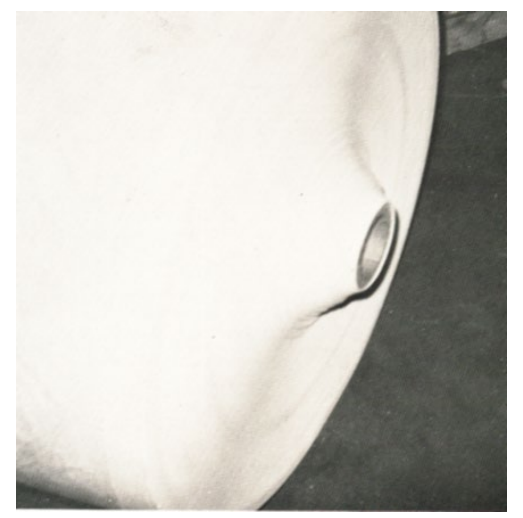

Figure 4 - Type 1B (Unwinding) Telescope 

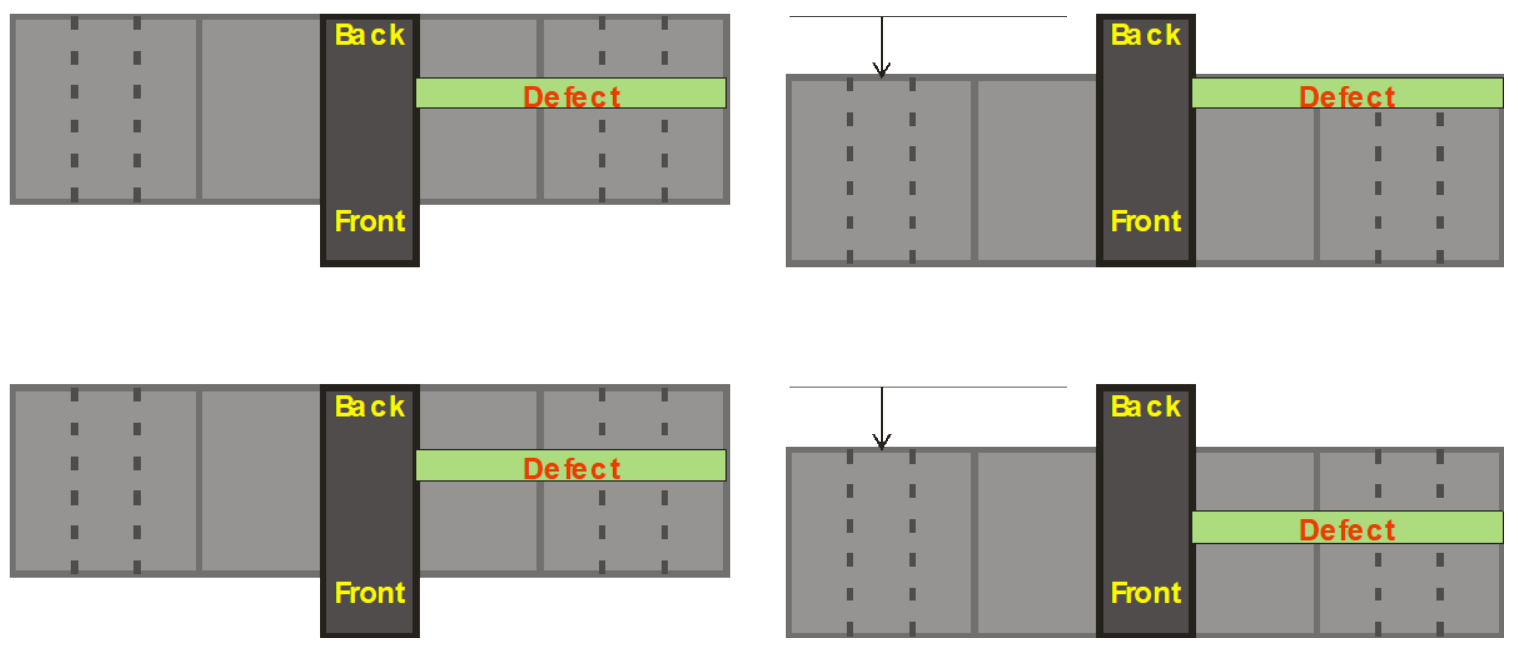

Figure 5 - One Way to Determine Whether it is Machine or Material 